\title{
Weak polarization-optical responses of diluted magnetic nanofluid probed by laser radiation with polarization modulation
}

\author{
Ya. A. Fofanov ${ }^{1}$, I. V. Pleshakov ${ }^{2}$, A. V. Prokof'ev ${ }^{2}$, E. E. Bibik ${ }^{3}$ \\ ${ }^{1}$ Institute for Analytical Instrumentation RAS, 26 Rizhsky ave., \\ St. Petersburg, 190103, box 207, Russian Federation \\ ${ }^{2}$ Ioffe Institute, 26 Politechnicheskaya str., St. Petersburg, 194021, Russian Federation \\ ${ }^{3}$ St. Petersburg State Institute of Technology (Technical University), \\ 26 Moskovsky ave., 190013, St. Petersburg, Russian Federation \\ ivanple@yandex.ru
}

PACS 78.20.Ls; 75.50.Mm; 42.60.-v

DOI 10.17586/2220-8054-2021-12-1-60-64

\begin{abstract}
A sensitive laser registration of weak polarization-optical responses was used for the investigations of dilute magnetic nanofluids. Criteria for weak and strong signals for probing of sample by laser radiation with deep modulation of polarization were considered. The magneto-optical responses of a kerosene-based fluid with magnetite nanoparticles were investigated over a wide (five orders of magnitude) range of concentrations. Weak polarization responses for this nanofluid were observed at record low volume concentrations of nanoparticles up to $10^{-7}$.
\end{abstract}

Keywords: magnetic nanofluid, laser probing, magnetooptics, weak polarization-optical response.

Received: 4 February 2021

Revised: 15 February 2021

\section{Introduction}

Magnetic nanofluids have attracted the attention of researchers to this day, and the particular interest in them is associated with the emergence of a number of new proposals for their application in optoelectronics, biomedicine, and other areas [1]. The main properties of these materials were established several decades ago [1-5], however they cannot be considered quite definitively studied. At present, magnetic nanofluids continue to be investigated from different points of view and by different methods, among which, optical analyses take an important place [1-5].

Approaches based on the use of light polarization are very informative and successfully complement magnetometric experiments [2-5]. In particular, the use of polarization modulation seems to be promising [6], and this has already been demonstrated in the study of different objects [7-12], although as applied to magnetic nanofluids the advantages of this method were not widely used.

Nonstationary laser polarization-optical probing in the original combination with a differential photodetection scheme allows one to achieve a very high sensitivity of optical measurements up to a threshold that is limited only by natural (quantum, photonic) noise of radiation [13-15]. Earlier, by the methodology developed on this basis, we investigated the polarization characteristics of various objects, including optical elements with a high degree of optical and structural homogeneity [16], magnetically ordered materials [17], and perfect optical crystals, including those with a modified crystal structure [15]. A preliminary polarization-optical diagnostics of magnetic fluids was also performed [18]. This paper presents the results of a study of weak polarization responses obtained for a wide range of concentrations in experiments with magnetic nanofluid probing by laser radiation with polarization modulation.

\section{Experimental details}

\subsection{Setup}

A simplified diagram of the experimental setup adapted for the study of magnetic nanofluids is shown in Fig. 1 (see also [16-18]). Probing laser radiation $(\lambda=0.63 \mu)$ passes through a polarization modulator, a cuvette with a magnetic nanofluid, and enters a polarization analyzer, where polarization-sensitive optical detection and preliminary processing of the photosignal performed, and then the signal enters the registration system and the computer. A cuvette with a magnetic fluid is placed in a transverse magnetic field $H$, which is created by solenoids. This field is linearly swept at values ranging from -45 to +45 Oe.

The analytical signal of the experimental setup, i.e., the observed polarization-optical response $\varphi$ can be represented as follows: $\varphi=\varphi_{0} \sin \Delta$. Here, $\varphi_{0}$ is a scale factor that takes into account, in particular, the degree of polarization modulation; $\Delta$ is the phase difference of two orthogonal linearly polarized components of the probing 


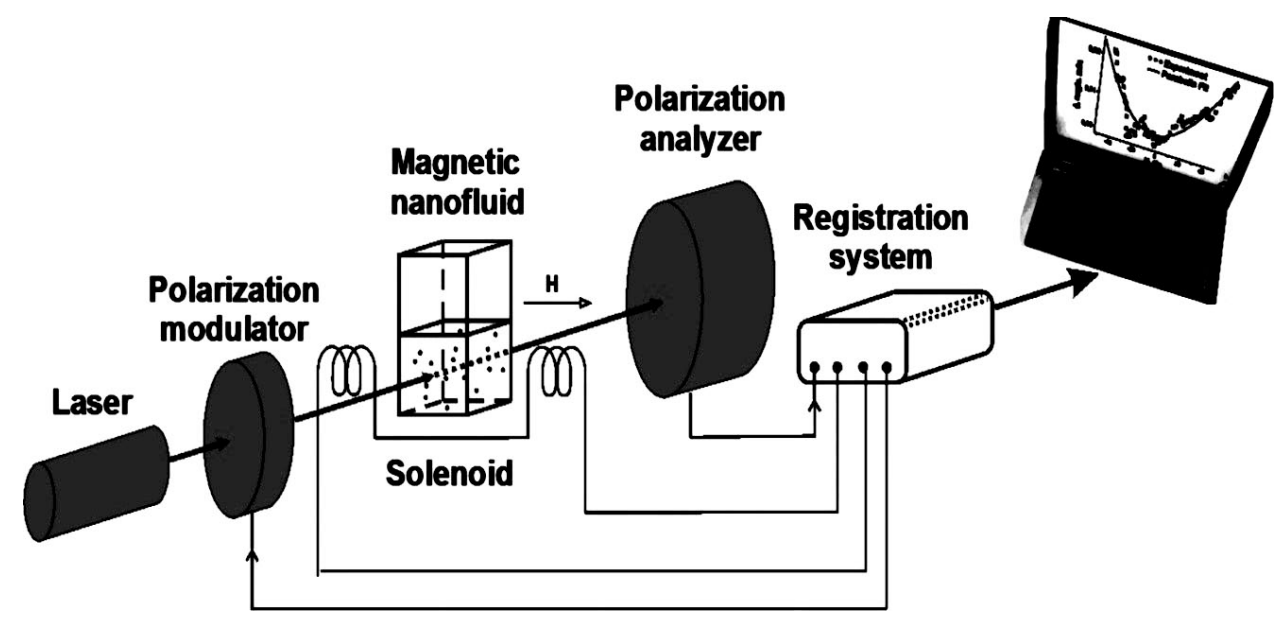

FIG. 1. Experimental setup

light arising as a result of passing through the object under study. Thus, the measured quantity is birefringence (linear optical anisotropy) of the magnetic fluid induced by an external magnetic field [16-18]. Further, we assume that the scale of the output signals is calibrated in units of the measured anisotropy (in our case, in angular minutes). A detailed description of the measurement and calibration procedures is given in [16-18].

\subsection{Weak polarization responses}

In a number of practical cases it is important to investigate samples with small optical anisotropy [16-19]. Our proposed natural criterion for the smallness of anisotropy is based on the inequality $\Delta \ll 1 \mathrm{rad}$ [14]. When it is so, $\sin \Delta \sim \Delta$ and the dependence $\varphi(\Delta)$ becomes linear. The degree of linearity of the function $\varphi(\Delta)$ depends on how well this inequality is fulfilled. In the opposite case, when $\Delta>1 \mathrm{rad}$, the anisotropy under study should be considered strong.

Polarization responses $\varphi$ corresponding to weak anisotropy $\Delta$ might also be called weak signals (weak responses), in spite of this, the output signals can be quite large in absolute terms. Except to linearity, the region of weak signals has another very useful property - the additivity of responses: the total response of a system of samples $\varphi_{\Sigma}$ is equal to the sum of their individual responses $\varphi_{i}: \varphi_{\Sigma}=\Sigma_{i} \varphi_{i}[16]$.

The threshold value of the measured birefringence $\Delta_{t h}$, determined by the natural noise of the photo registration process, is rather small and, according to the criterion considered above, is in the area of weak responses. Thus, for the power of the used laser is on the order of $1 \mathrm{~mW}$, the value of $\Delta_{t h}$ is about $1.5 \cdot 10^{-4} \mathrm{ang}$. min. (respectively, the value of $\varphi_{t h}$ is also about $1.5 \cdot 10^{-4}$ ang. min.) $[13,14,19,20]$. In practice, the minimum value of the recorded response can often be limited, for example, by fluctuations of the laser radiation parameters used and other instrumental factors, which requires careful organization of the measuring procedure [17,18,21].

To register weak polarization responses, we have provided the ability of precise adjustment of the transmission coefficient of the optoelectronic path in the range of more than three orders of magnitude with retention the calibration. Also, the accumulation of the signal during the implementation of series of multiple, up to twenty times, scanning of the magnetic field was provided.

\subsection{Samples}

The investigation was carried out on the kerosene-based magnetite solutions, prepared by the technique, described in [22] (the samples were the same as was used in [18,23]). The solid phase, dispersed in carrying fluid consisted of $\mathrm{Fe}_{3} \mathrm{O}_{4}$ particles with a characteristic size of about $10 \mathrm{~nm}$, coated by a surfactant (oleic acid). It is well known that at such sizes, ferrimagnetically ordered magnetite particles are single-domain and, in total, represent a system of independent magnetic moments that can be affected by an external magnetic field $[1,3]$. Thus, this two-component liquid (solid phase and solvent) is a nanostructure with magnetic properties. An important manifestation of the latter is that under the field action, this magnetic nanostructure takes on an optical anisotropy. This phenomenon of forming the optically anisotropic medium becomes possible due to the fact that magnetic particles themselves have a shape anisotropy and they can also accumulate into elongated agglomerates [2-4].

The material was a colloid synthesized by hydrolysis of iron chloride and sulfate solutions with ammonia followed by extraction of magnetite nanoparticles with a solution of oleic acid. This technology makes it possible to obtain a 
ferrofluid containing particles with a surface covered with a layer of surfactant (the thickness of which is much less than the diameter of their magnetic core) and an easily determined volume concentration of the solid phase [22].

Our experiments on observing field-induced birefringence started from the sample with concentration of $1 \cdot 10^{-2}$ (which ensured sufficient transparency of the nanofluid in a $1 \mathrm{~cm}$-thick cuvette), with its further successive dilutions up to $1 \cdot 10^{-7}$.

\section{Experimental results}

In Fig. 2 the dots show the measured polarization response vs $H$, for the nanofluid with initial concentration. To obtain a quantitative estimate of its value, a parabolic approximation was applied, which is shown in figure by the solid line. As one can see, it gives a fairly good agreement with the experiment, although there are some minor deviations. (A more detailed analysis of the similarity of the observed functions $\varphi(H)$, as well as a comparative analysis of their analytical approximations over a range of three orders of magnitude on concentration were carried out in $[18,24,25]$.) Fig. 3 depicts the similar dependencies for the sample with minimal investigated concentration.

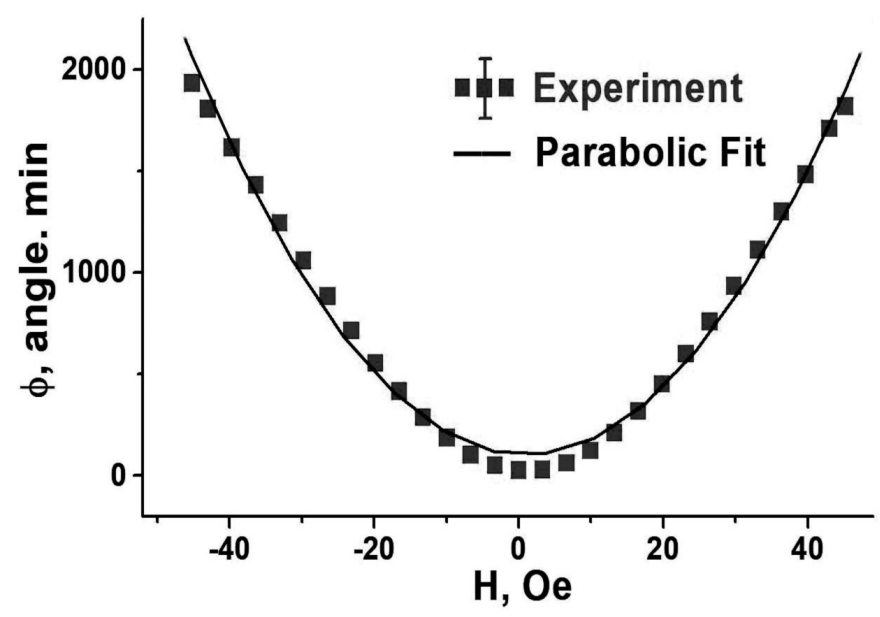

FIG. 2. Polarization responses for the concentration of $1 \cdot 10^{-2}$

The scatter of experimental data in this case can be explained by instrumental factors and, probably, by intrinsic fluctuations in the studied nanofluid.

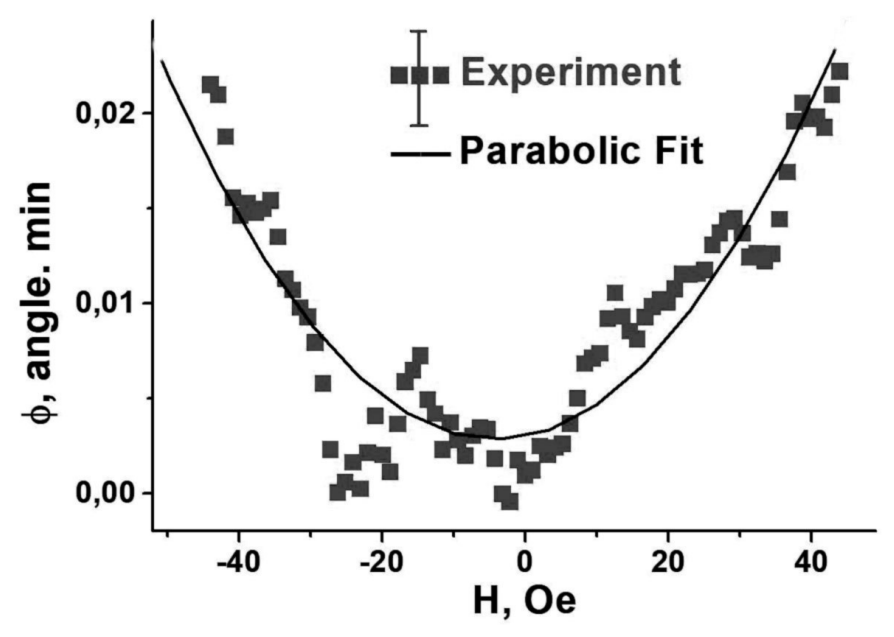

FIG. 3. Polarization responses for the concentration of $1 \cdot 10^{-7}$

From the obtained results, it is obvious that the value of parabolic fitting function taken at some fixed $H$ can be regarded as magnitude of the response $\varphi$ (further we used $H=43$ Oe). In Fig. 4 the dependence of $\varphi$ on concentration is shown; the figure also shows it is a linear approximation. Note that the measurement at the end of the range with minimal concentration indicates a very high sensitivity of the method used. 


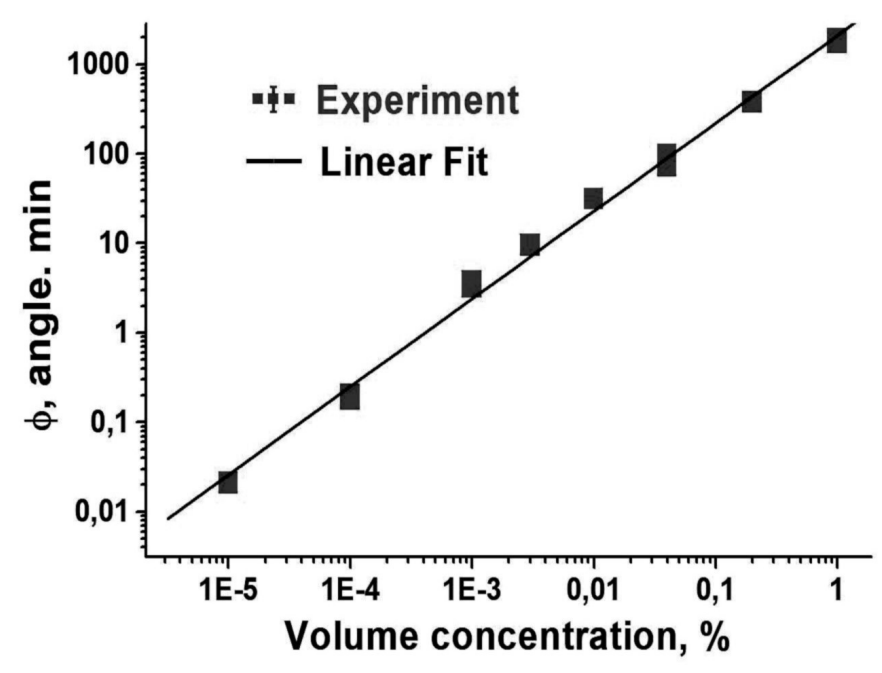

FIG. 4. Polarization response versus concentration of magnetic nanoparticles $(H=43 \mathrm{Oe})$

\section{Discussion}

The similarity of $\varphi(H)$ functions over a very wide range suggests the physical mechanism of birefringence in a magnetic nanofluid to be generally the same for both relatively large $\left(10^{-2}\right)$ and very small $\left(10^{-7}\right)$ concentrations of solid phase. At low concentrations, the nature of polarization responses cannot be entirely determined by the aggregates of nanoparticles induced by the field (we do not exclude, however, that this effect to some extent is present). This means, that for large concentrations, this mechanism may not be the only one. (In more detail, this fact is discussed in $[18,24,25])$.

Frequently used model for the birefringence in magnetic nanofluids is based on the idea of the orientation of individual nanoparticles in magnetic field [1-4]. In the theory developed in [2,4] the total anisotropy of the fluid is determined by the sum of contributions from individual non-spherical particles, taking into account their orientation with respect to the field direction. Such a model results in linear dependence of $\Delta$ on the number of magnetic particles.

This concept is in accordance with the linearity of the experimentally observed dependence $\varphi(\Delta)$ and with additivity of weak polarization responses for magnetic nanofluid under study (see also [18, 24, 25]). It is possible that up to those concentrations that in this work are considered as high, the influence of aggregates is still small. Further clarification of the proportion between contributions from aggregates and individual particles can probably be obtained by studying the dynamic characteristics of the investigated medium.

\section{Conclusion}

Our experiments have demonstrated a very high potency of the probing a magnetic nanofluid by laser radiation with polarization modulation. This way made it possible to detect the optical responses for a record low concentration of the solid phase $\left(10^{-7}\right)$.

It was found that over a very wide range of changes in the concentrations of the solid phase in nanofluids, at low magnetic fields $(H<45 \mathrm{Oe}$ ), birefringence is determined by one dominant mechanism, which is most likely associated with the anisotropy of the particle's shape.

In our opinion, the approach used in this work can be extended further, for example, to magnetic fluids modified by different additives, to impurity centers and defects in solids, biological tissues and others.

This work was performed in the framework of State tasks 075-01073-20-00 on the topic No. 0074-2019-0007 of the Ministry of Science and Higher Education of the Russian Federation.

\section{References}

[1] Scherer C., Figueiredo Neto A.M. Ferrofluids: Properties and applications. Braz. J. Phys., 2005, 35(3A), P. $718-727$.

[2] Skibin Yu.N., Chekanov V.V., Raker Yu.L. Birefringence in a ferromagnetic liquid. Sov. Phys. JETP, 1977, 45(3), P. 496-499.

[3] Davis H.W., Llewellyn J.P. Magnetic birefringence of ferrofluids: I. Estimation of particle size. J. Phys. D: Appl. Phys., 1979, 12(2), P. 311319.

[4] Scholten P.C. The origin of magnetic birefringence and dichroism in magnetic field. IEEE Trans. Magn., 1980, 16(2), P. 221-225.

[5] Zhao Y., Lv R., Zhang Y., Wang Q. Novel optical devices based on the transmission properties of magnetic fluid and their characteristics. Opt. Lasers Eng., 2012, 50(9), P. 1177-1184. 
[6] Badoz J., Billardon M., Canit J.C., Russel M.F., Sensitive devices to determine the state and degree of polarization of a light beam using a birefringence modulator. J. Opt., 1977, 8(6), P. 373-384.

[7] Jasperson S.N., Schnatterly S.E. An improved method for high reflectivity ellipsometry based on a new polarization modulation technique. Rev. Sci. Instrum., 1969, 40(6), P. 761-767.

[8] Shindo Y., Mizuno K., Sudani M., Hayakawa H., Ohmi Y., Sakayanagi N., Takeuchi N. New polarization-modulation spectrometer for simultaneous circular dishroism and optical rotatory dispersion measurements. II. Design, analysis, and evaluation of prototype model. Rev. Sci. Instrum., 1989, 60(12), P. 3633-3639.

[9] Acher O., Bigan E., Drevillon B. Improvements of phase-modulated ellipsometry. Rev. Sci. Instrum., 1989, 60(1), P. 65-77.

[10] Gupta V.K., Kornfield J.A., Ferencz A., Wegner G. Controlling molecular order in "Hairy-rod" Langmuir-Blodgett films: A polarizationmodulation microscopy study. Science, 1994, 265(5174), P. 940-942.

[11] Shindo Y., Kani K., Horinaka J., Kuroda R., Harada T. The application of polarization modulation method to investigate the optical homogeneity of polymer films. J. Plast. Film Sheeting, 2001, 17(2), P. 164-183.

[12] Osborn K.D., Singh M.K., Urbauer R.J.B., Johnson C.K. Maximum-likelihood approach to single-molecule polarization modulation analysis. ChemPhysChem, 2003, 4(9), P. 1005-1011.

[13] Fofanov Ya.A. Threshold sensitivity in optical measurements with phase modulation. Proceedings of the Conference. Tenth All-Union Symposium and School on High-Resolution Molecular Spectroscopy. Proc. SPIE, 1992, 1811, P. 413-414.

[14] Fofanov Ya.A., Sokolov I.M., Pleshakov I.V., Vetrov V.N., Prokofiev A.V., Kuraptsev A.C., Bibik E.E. On the criteria for strong and weak polarization responses of ordered objects and systems. Proceedings of the XI International Symposium on Photon Echo and Coherent Spectroscopy (PECS-2017), Svetlogorsk (Russia), 2017, EPJ Web Conf., 161, P. 01003.

[15] Fofanov Ya., Vetrov V., Ignatenkov B. Laser polarization-optical sounding of optical crystals and ceramics. Proceedings of 2018 International Conference Laser Optics (ICLO), St. Petersburg, 2018, P. 406-406,

[16] Sokolov I.M., Fofanov Ya.A. Investigations of the small birefringence of transparent objects by strong phase modulation of probing laser radiation. J. Opt. Soc. Am. A., 1995, 12(7), P. 1579-1588.

[17] Fofanov Ya.A., Pleshakov I.V., Kuz'min Yu. I. Laser polarization-optical detection of the magnetization process of a magnetically ordered crystal. J. Opt. Technol., 2013, 80(1), P. 64-67.

[18] Fofanov Ya.A., Pleshakov I.V., Prokof'ev A.V., Bibik E.E. Investigation of polarization magnetooptic responses of a low-concentration ferrofluid. Tech. Phys. Lett., 2016, 42(10), P. 1054-1056.

[19] Fofanov Ya.A. Nonlinear and fluctuation phenomena under conditions of strong selective reflection in inclined geometry. In Advances in Optoelectronics Research. Nova Science Publishers, USA, 2014, P. 75-114.

[20] Klyshko D.N., Masalov A.V. Photon noise: observation, squeezing, interpretation. Phys.-Uspekhi, 1995, 38(11), 1203-1230.

[21] Sokolov I.M., Fofanov Ya.A. Suppression of excess noise of polarization-modulated probe light in measurements of weak optical birefringence. Optics and spectroscopy, 1999, 86(5), P. 745-752.

[22] Bibik E.E., Matygullin B.Ya., Raikher Yu.L., Shliomis M.I. Magnetostatic properties of magnetite colloids. Magnetohydrodynamics, 1973, 9(1), P. 58-62.

[23] Prokof'ev A.V., Pleshakov I.V., Bibik E.E., Kuz'min Yu.I. An optical investigation of the geometric characteristics of aggregates formed by particles of magnetic fluid. Tech. Phys. Lett., 2017, 43(2), P. 194-196.

[24] Fofanov Ya.A., Manoilov V.V., Zarutskii I.V., Kuraptsev A.S. Statistical analysis of the data of highly sensitive laser polarization-optical probing of magnetic nanofluids. J. Opt. Technol., 2020, 87(2), P. 94-99.

[25] Fofanov Ya.A., Manoylov V.V., Zarutskiy I.V., Kuraptsev A.S. Laser polarization-optical diagnostics of ordered objects and structures. Bull. Russ. Acad. Sci.: Phys., 2020, 84(3), P. 263-266. 